\title{
UNA APROXIMACIÓN A LAS DESCARGAS POR EVAPOTRANSPIRACIÓN DEL ACUÍFERO FREÁTICO PAMPEANO EN LA CUENCA DEL ARROYO DEL AZUL (LLANURA PAMPEANA)
}

\section{AN APPROACH TO THE EVAPOTRANSPIRATION DISCHARGES OF THE PAMPEANO AQUIFER IN THE DEL AZUL BASIN (PAMPEAN PLAIN)}

\author{
Romina Marcovecchio $^{(1)(2)}$ y Marcelo Varni( ${ }^{(1)(3)(*)}$ \\ (1) Instituto de Hidrología de Llanuras. Azul, Buenos Aires, Argentina. \\ (2) Consejo Nacional de Investigaciones Científicas y Técnicas. Argentina. \\ (3) Universidad Nacional del Centro de la Provincia de Buenos Aires. Rectorado y Facultad de Ciencias \\ Humanas, Buenos Aires, Argentina. \\ ${ }^{(*)}$ e_mail: marcelovarni@gmail.com
}

\begin{abstract}
RESUMEN
Se estudian las recargas mensuales totales, las descargas por flujo subterráneo a zonas de descarga y las pérdidas por evapotranspiración directa desde el nivel freático a partir de la variación diaria del nivel freático en una perforación somera en la zona llana de la cuenca del arroyo del Azul, ubicada en la llanura pampeana. Los niveles freáticos se registraron mediante un sensor de presión piezorresistivo. El acuífero freático analizado es el acuífero Pampeano. Los niveles freáticos variaron en el período analizado (2007-2018) entre 0.5 y 4 m de profundidad. Se ha encontrado que las descargas por evapotranspiración directa desde el nivel freático son una fracción relevante de la recarga total (42\%). Las descargas por evapotranspiración directa son mayores en los meses cálidos, aunque la menor variabilidad interanual se da en los meses fríos y en los meses cálidos. La mayor variabilidad se da en otoño y primavera. En el período 2007-2018 la recarga total anual promedio fue de $252 \mathrm{~mm}$, la pérdida anual promedio de la recarga a la atmósfera fue de $105 \mathrm{~mm}$, con una desviación estándar de $25 \mathrm{~mm}$ y un coeficiente de variación de 0.24. Este porcentaje importante de la recarga total que se pierde por evapotranspiración directa hace que se deban considerar con especial atención los balances de agua en el suelo para estimar la recarga al acuífero (que no consideran esta pérdida) y explica ciertas incongruencias con los modelos de flujo de aguas subterráneas que exigían una disminución de la recarga en la zona más llana para posibilitar el ajuste de los niveles freáticos.
\end{abstract}

Palabras clave: recarga total al acuífero freático, descarga por flujo subterráneo, descarga directa por evapotranspiración

\begin{abstract}
Total monthly recharges, discharges by groundwater flow to discharge zones and losses by direct evapotranspiration from the water table are studied from the daily variation of the water table level in a shallow well in the flat area of the Azul stream basin., located at the Pampean plain. The phreatic levels were recorded using a piezoresistive pressure sensor. The analyzed phreatic aquifer is the Pampeano aquifer. The phreatic levels varied in the period analyzed (2007-2018) between 0.5 and $4 \mathrm{~m}$ deep. Direct evapotranspiration discharges from the water table have been found to be a relevant fraction of the total recharge (42\%). Direct evapotranspiration discharges are higher in warm months, although the least interannual variability occurs in cold and warm months. The greatest variability occurs in autumn and spring. In the period 2007-2018, the average annual total recharge was $252 \mathrm{~mm}$, the average annual loss of recharge to the atmosphere was $105 \mathrm{~mm}$, with a standard deviation of $25 \mathrm{~mm}$ and a coefficient of variation of 0.24 . This important percentage of the total recharge that is lost by direct evapotranspiration means that the soil water balances must be considered with special attention to estimate the recharge to the aquifer (which does not consider this loss) and explains certain inconsistencies with the groundwater flow models. that required a decrease in recharge in the flattest area to enable the adjustment of the water table levels.
\end{abstract}

Keywords: groundwater total recharge, groundwater flow discharge, direct discharge by evapotranspiration 


\section{INTRODUCCIÓN}

El área de estudio se ubica en la Pampa Húmeda argentina, con altitudes entre 240 y $70 \mathrm{msnm}$. Se trata de una zona de gran importancia desde el punto de vista económico debido a las características de su clima y sus suelos, con una excelente aptitud para el desarrollo de actividades agrícolas y ganaderas. La actividad agrícola se basa en los cultivos de trigo, maíz, soja, cebada, centeno, lino y girasol, así como la papa. También se desarrollan en la región actividades pecuarias de cría de ganado (bovino y ovino), y también la lechería.

El área concreta es la cuenca del arroyo de Azul (Figura 1). El curso nace en cercanías de la localidad de Chillar y transporta sus aguas hacia el norte, hasta perderse en la llanura antes de alcanzar el río Salado. En la zona llana más baja, a unos $80 \mathrm{~km}$ al norte de la ciudad de Azul, el curso es interceptado y drenado parcialmente por un canal artificial, el cual se adopta en este trabajo como límite norte del área de estudio. La cuenca está integrada por una gran planicie al norte y un área más pequeña de suaves serranías al sur, y ambas están conectadas a través del piedemonte. Estos tres sectores podrían considerarse esquemáticamente como subcuencas: alta (serranías), intermedia (piedemonte) y baja (llanura). La pendiente media del terreno es del 5\% en la zona alta, entre 0.5 y $0.8 \%$ en la intermedia y $0.2 \%$ en la baja. El comportamiento global de la región es el de una llanura (Sala et al., 1987).

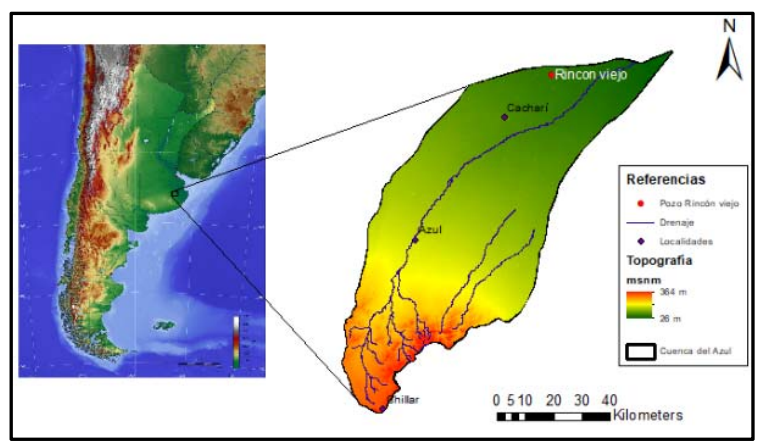

Figura 1. Ubicación del área de estudio y del pozo analizado.

En las serranías ubicadas al sur existen afloramientos rocosos compuestos por rocas metamórficas, tonalitas, migmatitas y cuarcitas (González Bonorino et al., 1956). Dichas rocas se profundizan hacia el norte, constituyendo la base del sistema hidrológico, y alcanzan una profundidad cercana a los $800 \mathrm{~m}$ al noreste del área de estudio. En la ciudad de Azul (altitud de $140 \mathrm{msnm}$ ) dicho basamento se ha detectado a unos $120 \mathrm{~m}$ de profundidad. Encima del basamento cristalino yacen sedimentos loéssicos que contienen el acuífero freático Pampeano (Sala et al., 1983), que está constituido por arenas limosas y limos arcillosos con mayor o menor presencia de arcillas. Estos sedimentos muestran cierta heterogeneidad vertical y poca continuidad espacial. La Formación Post-Pampeano (Fidalgo et al., 1975) se ubica por encima del Pampeano, y a efectos prácticos se pueden reunir en una sola unidad hidrogeológica.

En las cuencas alta e intermedia la conductividad eléctrica de las aguas subterráneas presenta valores menores a $750 \mu \mathrm{S} / \mathrm{cm}$, aunque pueden observarse leves incrementos en algunos sectores aledaños a los cursos de agua, donde se produce la descarga parcial de aguas subterráneas más profundas, asociadas a un mayor recorrido y tiempo de renovación. En la cuenca baja existe un aumento gradual de salinidades hasta valores de $3000 \mu \mathrm{S} / \mathrm{cm}$. Desde el punto de vista químico las aguas son preponderantemente bicarbonatadas, con predominio cálcico en cabeceras, pasando a dominar las sódicas en el sentido del flujo, para terminar con incremento de los contenidos de sulfatos y cloruros al final de la cuenca.

La estación con registros climáticos más prolongados en la cuenca es la estación Azul Aero, perteneciente al Servicio Meteorológico Nacional. En ella la precipitación (P) media anual es de 911 mm (19012019) y la temperatura media anual es $14.4^{\circ} \mathrm{C}$ (19662019). Los meses menos lluviosos son Junio, Julio y Agosto (45, 45 y $46 \mathrm{~mm}$, respectivamente) mientras que los más lluviosos son Enero, Febrero y Marzo (93, 90 y $118 \mathrm{~mm}$, respectivamente). De acuerdo con la clasificación de Thornthwaite, el clima de la región es subhúmedo-húmedo, mesotermal, con pequeño a nulo déficit de agua.

El objetivo del presente trabajo es lograr una aproximación de la evapotranspiración desde el acuífero freático en la cuenca baja, debido a que se considera que la evapotranspiración llega a tener magnitudes importantes en zonas de llanura como las que aborda el presente estudio. La evaporación desde un nivel freático somero es un proceso importante para los balances de agua y sales. En este proceso son relevantes dos mecanismos: evaporación desde la superficie del terreno sostenida por un flujo líquido continuo sostenido desde el nivel freático, y evaporación subsuperficial mantenida por la remoción de vapor difusivo a través del medio poroso (Assoulineet al., 2013). Miao et al. (2017) analizan cómo procesos de 
congelamiento y descongelamiento afectan la evaporación desde niveles freáticos someros. van Dam y Feddes (2000) analizan tanto la infiltración como la evaporación y los niveles freáticos resultantes a través de simulación numérica. Menking et al. (2000) estudiaron la evaporación desde niveles freáticos someros, pero en clima árido. Zhang et al. (2001) analizaron este proceso derivando ecuaciones a partir de mediciones en lisímetros. En nuestro caso, se ha aproximado este valor a partir de registros de niveles freáticos obtenidos en un pozo de observación somero localizado en la zona llana de la cuenca.

\section{METODOLOGÍA}

El Instituto de Hidrología de Llanuras posee una red de pozos someros de monitoreo instalados a lo largo y ancho de la cuenca del arroyo del Azul. Los mismos poseen limnígrafos digitales con sensor piezoresistivo para el registro del nivel freático. Este trabajo analiza los datos diarios de la perforación Rincón Viejo, ubicada en la parte baja de la cuenca, entre los años 2007 y 2018.

La recarga es el agua que infiltra a través de la zona no saturada hasta el nivel freático $\mathrm{y}$, por lo tanto, aumenta el volumen de agua almacenada en la zona saturada (Lerner et al., 1990; Healy y Cook, 2002; Scanlon et al., 2002). Se utilizó en este estudio el método de fluctuación del nivel freático (WTF)para el cálculo de la recarga mensual, a partir de datos diarios. Este método principalmente es aplicable a acuíferos freáticos (Risser et al., 2005).

Las mediciones frecuentes y precisas del nivel del agua disponibles se encuentran en un área no afectada por actividades humanas. El método es muy útil para detectar variaciones de recarga con una discriminación temporal detallada (Varni et al., 2013). Se basa en la premisa de que el aumento de los niveles de agua subterránea se debe a la recarga de agua que llega a la capa freática. Suponiendo que la cantidad de agua disponible en una columna de agua, es igual al rendimiento especifico (Sy) multiplicado por la altura de la columna de agua, siendo entonces posible el cálculo de la recarga a partir de (Healy y Cook 2002; Varni et al., 2013):

$$
\mathrm{R}=\mathrm{S}_{\mathrm{y}} \cdot \frac{\Delta \mathrm{h}}{\mathrm{t}}
$$

Un desafío del método WTF implica la estimación del rendimiento específico (Sy) del acuífero a la profundidad donde se produce la fluctuación del nivel freático. Para estimar Sy, se propuso un procedimiento gráfico, para el cual, se traza un gráfico de dispersión de los valores de precipitación (abcisas) frente a las elevaciones del nivel freático (ordenadas) evento a evento precipitación-recarga. La inversa de la pendiente de una línea dibujada a través del origen hasta justo por encima de todos los puntos medidos proporciona el valor máximo del rendimiento específico (Varni et al., 2013).

Se puede hacer una distinción entre recarga total, obtenida mediante la ecuación (1), y neta (Crosbie et al., 2005). La cantidad total de agua que llega a la capa freática es la recarga total, mientras que la recarga neta explica la posterior pérdida de agua de la zona saturada por evapotranspiración, o sea la recarga de agua que efectivamente queda finalmente en el acuífero freático. En áreas con nivel freático poco profundo, vegetación de raíces relativamente profundas, y/o suelos con materiales finos que produzcan una franja capilar muy desarrollada, esta pérdida de agua posterior puede ser considerable (Barua et al., 2020).

El cálculo de la recarga total mensual $R_{t}$ se calcula a partir de la sumatoria de ascensos diarios individuales de cada mes $\Sigma \Delta h i$. La recarga neta mensual menos la descarga por flujo subterráneo se obtiene de la diferencia de altura del nivel freático (Ah) entre los primeros días de meses consecutivos, considerando que esta diferencia de nivel incluye las recargas totales de eventos y las descargas por flujo subterráneo, evaporación y evapotranspiración ocurridas en el mes. Para el cálculo de la recarga total se utiliza la ecuación (1):

$\mathrm{R}_{\mathrm{t}}=\sum \Delta \mathrm{hi} \cdot S_{y}$

mientras que la recarga neta $R_{n}$ menos el flujo de descarga $q, R_{n-q}$ se obtiene de:

$\mathrm{R}_{\mathrm{n}-\mathrm{q}}=\Delta \mathrm{h}_{\mathrm{m}} \cdot \mathrm{S}_{\mathrm{y}}$

Finalmente, se obtiene la descarga mensual (D) de la diferencia entre (2) y (3):

$\mathrm{D}=\mathrm{R}_{\mathrm{t}}-\mathrm{R}_{\mathrm{n}-\mathrm{q}}$ 
La descarga tiene dos componentes: la descarga por flujo subterráneo $D_{q}$ y la descarga por evapotranspiración, la cual aumenta cuando el nivel freático se encuentra cerca de la superficie y cuando la atmósfera tiene una mayor demanda de agua.

Consideramos que durante los meses de temperaturas más bajas (junio, julio, agosto) la evapotranspiración es mínima. Así es que, simplificando, las descargas totales ocurridas en la estación fría suponemos que son fundamentalmente descargas por flujo.

Dada la vinculación de estas descargas con la altura del nivel freático, se analizó la relación existente entre la altura del nivel freático y las descargas producidas por flujo subterráneo durante los meses más fríos. Se ajustó una función exponencial decreciente de la forma:

$\mathrm{D}_{\mathrm{q}}=\mathrm{A} \cdot \mathrm{e}^{-\mathrm{Bh}}+\mathrm{C}$

debido a que las descargas por flujo disminuyen al aumentar la profundidad del nivel freático, como resultado de un nivel base de descarga que permanece prácticamente constante.

A través de la diferencia mensual entre las descargas totales $D$ obtenidas de la ecuación (4) y las descargas por flujo $D_{q}$ alcanzadas en la ecuación (5), se logró una aproximación al valor mensual de las descargas por evapotranspiración en la perforación de Rincón Viejo.

$$
\mathrm{EVT}=\mathrm{D}-\mathrm{D}_{\mathrm{q}}
$$

\section{RESULTADOS}

Se aproximó un valor de rendimiento especifico Sy mediante el método de Varni et al. (2013) (Figura 2) para la perforación de Rincón Viejo de 0.08, un valor coincidente con los valores teóricos de arcilla arenosa/ limo arenoso (Johnson, 1967).

Las recargas totales y netas obtenidas mensualmente muestran una variación estacional. Las recargas totales (procedentes de la precipitación) son en general mayores los meses de marzo y mayo, así como en agosto y septiembre. Esto coincide con la distribución de lluvias importantes (otoño y primavera). También puede haber lluvias considerables en verano, pero contribuyen principalmente a alimentar la evapotranspiración. A partir del año 2012 aumentan las recargas totales con respecto a los años anteriores, y se observa como acompaña a este aumento la disminución de la profundidad del nivel freático (Figura 3).

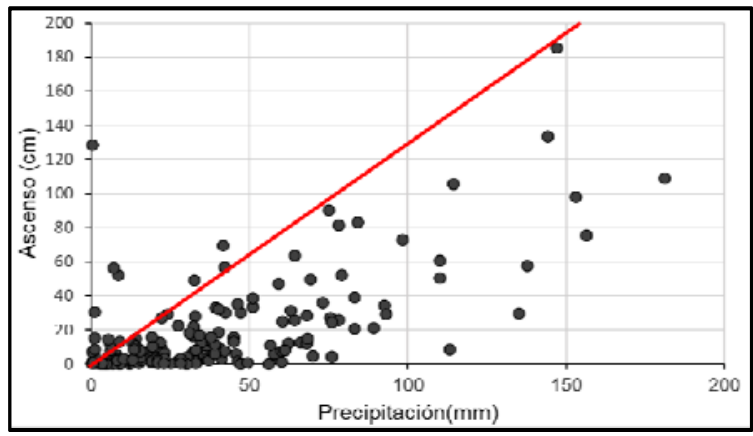

Figura 2. Estimación del rendimiento específico (Sy).

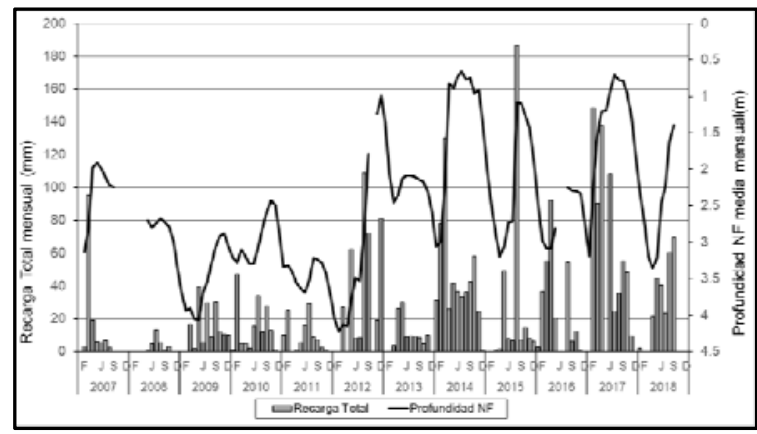

Figura 3. Variaciones mensuales de la recarga total y del nivel freático.

Del ajuste de la Ecuación (5) por mínimos cuadrados se obtuvo $A=82.5, b=-0.613$ y c=-5.06. El análisis de la ecuación obtenida de la relación entre la profundidad del nivel freático y las descargas ocurridas durante los meses de menores temperaturas anuales nos permite estimar que las descargas por flujo tienden a cero a medida que se acercan a una profundidad de 5 metros en la perforación Rincón Viejo (Figura 4).

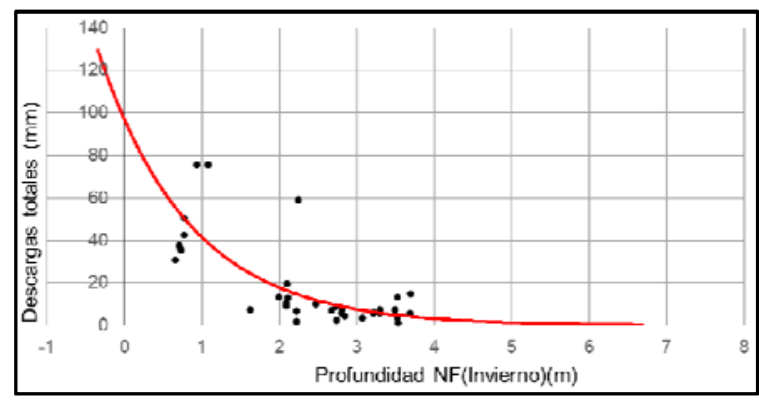

Figura 4. Relación entre la profundidad del nivel freático y las descargas totales en los meses de menores temperaturas entre 2007-2018 en Rincón Viejo. 
Con la curva ajustada en la Figura 4 se pueden obtener las descargas por flujo subterráneo para cada mes. Si a las recargas totales mensuales se restan estas descargas por flujo se obtienen las descargas por evapotranspiración.

\section{DISCUSIÓN}

La evaporación aumenta los meses cálidos y a medida que el nivel freático se acerca a la superficie. Aumentando de forma importante en los meses de mayores temperaturas cuando el nivel freático se encuentra a profundidades menores de los 2.50 metros.

El análisis de las descargas medias mensuales por evaporación entre los años 2007-2018 en Rincón Viejo nos muestra que las mayores magnitudes de evaporación ocurren los meses más cálidos del año; sin embargo, las estaciones de primavera y otoño presentan mayor variabilidad interanual en el período analizado (Figuras 5 y 6 ).

Los meses con mayores y menores temperaturas del año presentan menor variabilidad interanual de la evaporación, con valores de 24 mm mensuales en los meses de enero y diciembre, y de $0 \mathrm{~mm}$ en julio en la cuenca baja del arroyo del Azul (Figura 5).

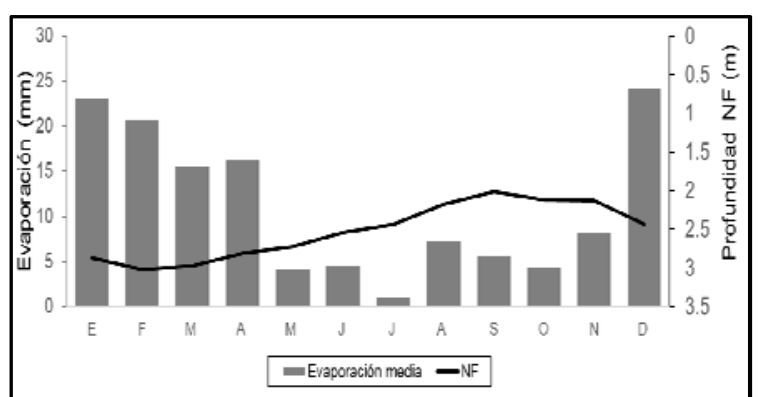

Figura 5. Descargas por evapotranspiración directa desde el nivel freático (2007-2018).
Los meses de marzo a agosto (la estación más fría del año) el nivel freático tiende a ascender y la recarga total media mensual presenta los mayores valores del año (Figura 6).

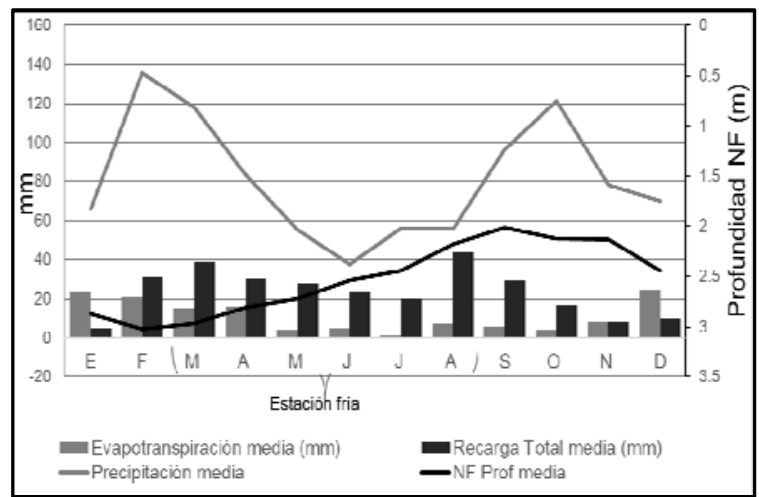

Figura 6. Evapotranspiración media mensual y recarga total media mensual (2007-2018).

Las descargas por evapotranspiración son mayores los meses temperaturas más altas (diciembre, enero, febrero); de modo que parece influir en la profundización del nivel freático (Figura 6).Se observa que los meses de transición entre las estaciones (febrero, marzo, abril, agosto, septiembre) presentan mayor variabilidad interanual tanto en las descargas por evapotranspiración como en las recargas totales (Figura 6).

En la Figura 7 puede verse el resultado de los siete años medidos en su totalidad: la recarga total, la descarga a la atmósfera de una parte de esta recarga y la profundidad de los niveles freáticos medios anuales. La recarga total anual promedio fue de $252 \mathrm{~mm}$, la pérdida anual promedio de la recarga a la atmósfera fue de $105 \mathrm{~mm}$ (42\% de la recarga total), con una desviación estándar de 25 mm y un coeficiente de variación de 0.24. 

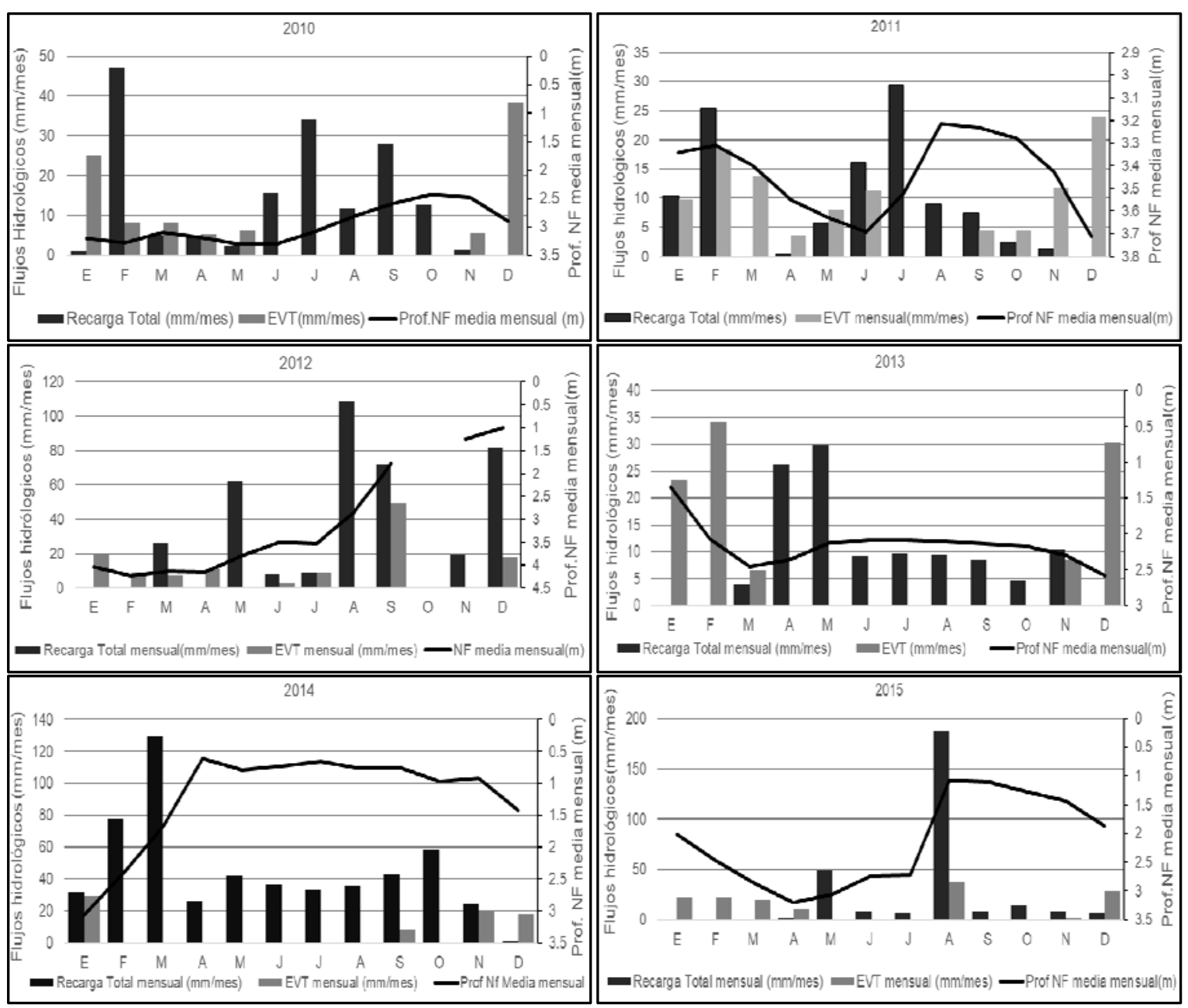

Figura 7. Recarga total y evapotranspiración desde el acuífero freático medias mensuales, en barras, y profundidad del nivel freático medio mensual en línea continua desde 2009 a 2015 inclusive.

\section{CONCLUSIONES}

Se analizaron las recargas totales mensuales a partir de datos diarios de nivel del agua subterránea por medio del método de fluctuación del nivel freático. Se estimaron las descargas mensuales por flujo hacia zonas de descarga a través del ajuste de una ecuación que relaciona el nivel en el pozo analizado en los meses invernales, que se suponen con pérdidas evaporativas nulas. Finalmente, se determinaron las descargas por evaporación y evapotranspiración desde el nivel freático, las cuales representan una pérdida importante de la recarga total (42\%).

En el período 2007-2018 la recarga total anual promedio fue de $252 \mathrm{~mm}$, la pérdida anual promedio de la recarga a la atmósfera fue de $105 \mathrm{~mm}$, con una desviación estándar de $25 \mathrm{~mm}$ y un coeficiente de variación de 0.24 .

\section{REFERENCIAS}

Assouline, S., Tyler, S.W., Selker, J. S., Lunati, I., Higgins, C. W. y Parlange, M. B. (2013). Evaporationfrom a shallow water table: diurnal dynamics of water and heat at the surface of drying sand. Water Resources Research, 49 (7) 4022-4034.

Barua, S., Cartwright, I., Dresel, P. E. y Daly, E.(2020). Using multiple methods to understand groundwater recharge in a semi-arid area, Hydrol. Earth Syst. Sci. Discuss., https://doi.org/10.5194/hess-2020-143, (in review).

Crosbie, R. S., Binning, P. y Kalma, J. D. (2005). A time series approach to inferring groundwater recharge using the water table fluctuation method. Water Resources Research, 41, W01008, doi:10.1029/2004WR003077.

Fidalgo, F., Pascual, R. y De Francesco, F. (1975). Geología superficial de la llanura Bonaerense 
(Argentina). Actas del VI Congreso Geológico Argentino, 103-138.

González Bonorino, R., Zardino, R., Figueroa, M. y Limousin, T. (1956). Estudio geológico de las Sierras de Olavarría y Azul (Bs. As.). LEMIT, Serie II (63), pp 5-22.

Healy, R. W. y Cook, P. G. (2002). Using groundwater levels to estimate recharge. Hydrogeology Journal 10(1): 91-109.

Johnson, A. I. 1967. Specific yield-compilation of specific yields for various materials. Hydrologic Properties of Earth Materials, U.S Geological Survey Water-supply Paper, 1662- D.

Lerner, D. N., Issar, A. S. y Simmers, I. (1990). Groundwater recharge. A guide to understanding and estimating natural recharge. IAH Int. Contrib. Hydrogeol. 8, Heinz Heise, Hannover, 345 pp.

Menking, K. M., Anderson, R. Y., Brunsell, N. A., Allen, B. D., Ellwein, A. L., Loveland, T. A.y Hostetler, S.W. (2000). Evaporation from groundwater discharge playas, Estancia Basin, central New Mexico. Global and Planetary Change 25:133-147.

Miao, Ch., Chen, J., Zheng, X., Zhang, Y., Xu, Y.y Du, Q. (2017). Soil water and phreatic evaporation in shallow groundwater during an freeze-thaw period. Water 9:396-408. doi: 10.3390/w9060396.

Risser, D. W., Gburek, W. J. y Folmar, G. J. (2005). Comparison of methods for estimating groundwater recharge and base flow at a small watershed underlain by fractured bedrock in the eastern United States (Vol. 5038). US Department of the Interior, US Geological Survey.

Sala, J. (1983). Generalizaciónhidrológica de la Provincia de Buenos Aires. Coloquio Intern. de Grandes Llanuras. Unesco. V III, Olavarria, Prov. de Buenos Aires, Argentina, 1983.

Sala, J. M., Kruse, E., y Aguglino, R. (1987). Investigaciónhidrológica de la cuenca del arroyo Azul, Provincia de Buenos Aires. CIC Informe 37.
Scanlon, B. R., Healy, R. W. y Cook, P. (2002). Choosing appropriate techniques for quantifying groundwater recharge. Hydrogeology Journal 10:18-39.

van Dam, J. C. y Feddes, R. A. (2000). Numerical simulation of infiltraton, evaporation and shallow groundwater levels with the Richards equation. Journal of Hydrology 223(1-4):72-85.

Varni, M., Comas, R., Weinzettel, P. y Dietrich, S. (2013). Application of water table fluctuation method to characterize the groundwater recharge in the Pampa plain, Argentina. Hydrological Sciences Journal 58(7): 1445-1455, doi: 10.1080/02626667.2013.833663.

Zhang, L., Dawes, W.R. y Walker, G.R. (2001). Response of mean anual evapotranspiration to vegetation changes at catchment scale. Water Resources Research 37(3):701-708.

\section{Tipo de Publicación: ARTICULO.}

Trabajo recibido el 09/09/2020 y aprobado para su publicación el 15/10/2020.

\section{COMO CITAR}

Marcovecchio, R. y Varni, M. (2020). Una aproximación a las descargas por evapotranspiración del acuífero freático pampeano en la cuenca del arroyo del Azul (Llanura Pampeana). Cuadernos del CURIHAM. 26: 13-19. DOI: https://doi.org/10.35305/curiham.v26i0.149

Este es un artículo de acceso abierto bajo licencia: Creative Commons Atribución - No Comercial Compartir Igual 4.0 Internacional (CC BY-NC-SA 4.0) (https://creativecommons.org/licenses/by-ncsa/4.0/deed.es) 